\title{
Two-Step Image Hallucination and Its Application to 3D Medical Image Super-Resolution
}

\author{
Yuto Kondo,X.H. Han \\ Graduate School of Information Science and Engineering, \\ Ritsumeikan University \\ Shiga, Japan
}

\author{
X. Wei \\ Institute for Infocomm Research, A-STAR \\ Singapore 138632
}

\author{
Y.W. Chen \\ Graduate School of Information Science and Engineering, Ritsumeikan University \\ Shiga, Japan \\ School of Health Sciences, Tokyo Metropolitan University \\ Tokyo, Japan
}

\begin{abstract}
In medical diagnosis, high resolution (HR) images are indispensable for giving more correct decision. However, in order to obtain high resolution medical images, it is necessary to impose long-time, hence it leads to heavy burden to the patient. Therefore Super Resolution technique, which can generate high resolution images from low resolution images using machine learning techniques, attracts hot attention recently. Therein, face hallucination is one of widely used super-resolution methods in image restoration field. However, the conventional face hallucination generally cannot recover high frequency information. Therefore, this paper integrates a further learning step into the conventional method, and proposes a 2-step image hallucination, which is prospected to recover most high frequency information lost in the available low-resolution input. Furthermore, we apply the proposed strategy to generate the high-resolution Z-direction data using self-similarity among different direction for 3D medical MR images. Experimental results show that the proposed strategy can reconstruct promising HR coronal or sagittal plane by using available LR and HR data pairs in axial plane.
\end{abstract}

Keywords-image restoration; super-resolution; medical volumetric image

\section{INTRODUCTION}

In medical imaging, the image resolution comes limited by a number of factors such as performance of hardware, time limitations or patient's comfort. In many cases, the acquired image resolution is too low to diagnosis. Super resolution image reconstruction (SR) is one of the techniques to solve theproblem[1]. SR is to generate a high resolution(HR) image from input low resolution(LR) image. SR can be broadly classified into two of methods.(1)the multi-frame SR[2,3] and (2)the single-frame SR [4,5]. Multi-frame SR is to reconstruct a HR image by combining multiple sub-pixel-warped LR images such as movie. Single-frame SR is to reconstruct a HR image from a LR image and database.

Face hallucination is one of single-frame SR methods [6-9], which is resolution enhancement of facial images. In this paper, we apply face hallucination to the case of medical image and propose a high-frequency compensation framework based on residual images for face hallucination method in order to improve the reconstruction performance.Furthermore, we propose new approach that reconstruct HR medical image. In medical imaging, we can obtain HR axial plane images however cannot obtain other direction HR images. On this problem, we apply the database that stored LR and HR data pairs in axial plane to reconstruct LR other plane images.The paper is organized as follows. In section 2, we describe the conventional methods. Section 3 describes our proposed method. Section 4 and 5 presents the experimental results and quantitative equation. Section 6 summarizes our conclusions.

\section{CONVENTIONAL Methods}

\section{A. Example-Based SR}

Example-Based SR is one of single-frame SR. It can be broadly classified into two of algorithm training step and test step. In training step, pairs of low LR and corresponding HR image patches are collected. These stored patches are called database. In the test step, each patch of the given LR image is compared to the LR patches in database, and the HR patch corresponding to the nearest LR patch is selected as the reconstructed HR image. In this paper we use frequency as the image feature vector. That is, pairs of medium frequency (MF) of image patches and high frequency (HF) of image patches are stored instead of LR and HR patches into database. Figure 1 shows the framework of the Example-Based SR. 


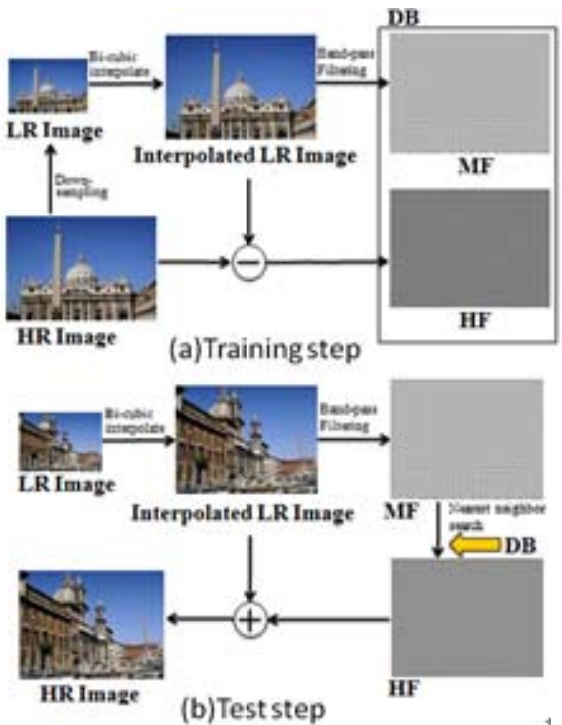

FIGURE I. THE FRAMEWORK OF THE EXAMPLE-BASED SR.

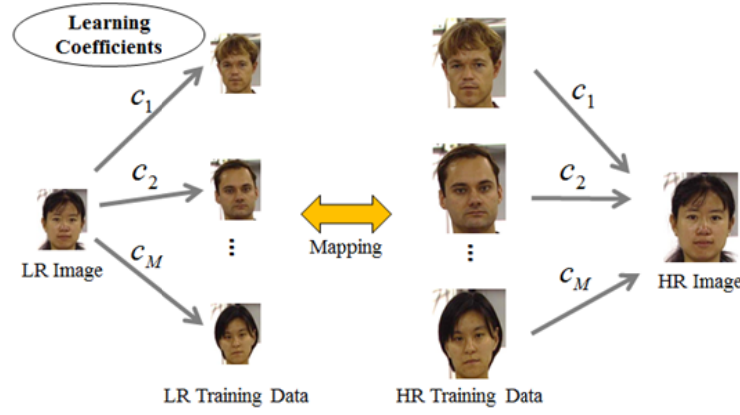

FIGURE II. THE BASIC CONCEPT OF FACE HALLUCINATION.

\section{B. Face Hallucination}

The face hallucination method is one of learning-based SR methods, which is proposed for resolution enhancement of facial images. In this section, we briefly introduce the basic concept of face hallucination, which is shown in Figure 2.The basic idea of face hallucination is that a face image can be reconstructed from other face images by linear combination because all facial images have a similar structure. In this method, each image patches are represented by a linear sum of learning data patches from a database. Therefore HR image can be reconstructed high quality even if database is small.

We represent a two-dimensional face image using a column vector of all pixel values.InputLR image patch $\mathbf{Y}_{L}$ is represented by a linear sum of $\left[\mathbf{L}_{1}, \mathbf{L}_{2}, \ldots, \mathbf{L}_{M}\right]$, where $M$ is the number of training patches.Similarly HR image patch $\mathbf{X}_{L}$ is represented by a linear sum of $\left[\mathbf{H}_{1}, \mathbf{H}_{2}, \ldots, \mathbf{H}_{M}\right]$.

$$
\begin{aligned}
\mathbf{Y}_{L} & =c_{1} \mathbf{L}_{1}+c_{2} \mathbf{L}_{2}+\cdots c_{M} \mathbf{L}_{M} \\
& =\sum_{i=1}^{M} c_{i} \mathbf{L}_{i}
\end{aligned}
$$

where $\mathbf{C}=\left[c_{1}, c_{2}, \cdots c_{M}\right]$ are the weight coefficients, satisfying equation (2). The optimal weights can be calculated by minimizing the error in reconstructing the input LR image $\mathbf{Y}_{L}$ from training LR image. This error is defined in (3).

$$
\begin{gathered}
C_{1}+C_{2}+\cdots C_{M}=1 \\
\varepsilon(\mathbf{C})=\left\|\mathbf{Y}_{L}-\sum_{i=1}^{M} c_{i} \mathbf{L}_{i}\right\|^{2}
\end{gathered}
$$

After substitution of the constraints in (2) into (3), the weight vector may be obtained using covariance matrix $\mathbf{S}$ in (4).

$$
\begin{gathered}
\mathbf{S}=\left(\mathbf{Y}_{L} \mathbf{E}^{T}-\mathbf{L}\right)^{T}\left(\tilde{\mathbf{Y}} \mathbf{E}^{T}-\mathbf{L}\right) \\
\mathbf{C}=\frac{\mathbf{S}^{-1} \mathbf{E}}{\mathbf{E}^{T} \mathbf{S}^{-1} \mathbf{E}}
\end{gathered}
$$

where $\mathbf{L}$ is a matrix combined by ordered each column vector in interpolated LR training patch $\mathbf{L}_{\mathbf{k}}, \mathbf{E}$ is a [ $M \times 1$ ] column vector in which all the elements are equal to 1 . After obtaining the coefficients for reconstructing the input LR image with LRtraining image as given (1),we replace $\left[\mathbf{L}_{1}, \mathbf{L}_{2}, \ldots, \mathbf{L}_{M}\right]$ with $\left[\mathbf{H}_{1}, \mathbf{H}_{2}, \ldots, \mathbf{H}_{M}\right]$ using the same coefficients $\mathbf{C}$. Subsequently, HR image $\mathbf{Y}_{H}$ can be obtained using following equation.

$$
\begin{aligned}
\mathbf{Y}_{H} & =c_{1} \mathbf{H}_{1}+c_{2} \mathbf{H}_{2}+\cdots c_{M} \mathbf{H}_{M} \\
& =\sum_{i=1}^{M} c_{i} \mathbf{H}_{i}
\end{aligned}
$$

\section{TWO-STEP IMAGE HALLUCINATION}

In this paper, we apply the face hallucination method to the case of a medical image. There is a reason that basic idea of face hallucination is that a face image can be reconstructed from other face images by linear combination because all facial images have a similar structure. Moreover medical image can be reconstructed from other medical images by linear combination as well as facial image. Therefore we apply face hallucination method to the case of a medical image. We call this method image hallucination in this paper.

Image hallucination is a useful technique however some detailed high-frequency components cannot be recovered. In this section, we propose a high-frequency compensation framework based on residual images for image hallucination method in order to improve the reconstruction performance.

We introduce the framework of proposed method. This method is separated into two algorithm, test step and training step. In the training step, we first obtain the LR image from the input HR image by down sampling. Next, we use image hallucination method to obtain a reconstruct image and calculate the LR residual image between the input interpolated LR image and the reconstructed HR image. Then we calculate the HR residual image between the HR image and the 
reconstruct image. That is the training step of 2-step image hallucination method, which is shown in left of figure 3.The goal of training step is to make residual database. In the test step, we first use image hallucination method to obtain a reconstruct image and calculate re the LR residual image between the interpolated input LR image and the reconstructed image. Then we use image hallucination method with using residual database to obtain the HR residual image. Finally we merge the HR residual image and the reconstruct HR image. That is the test step of 2-step image hallucination method, which is shown in right of figure 5.

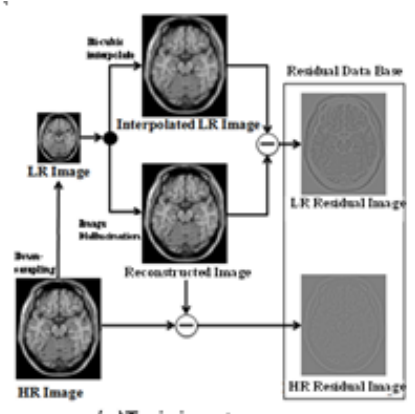

(a) Training step

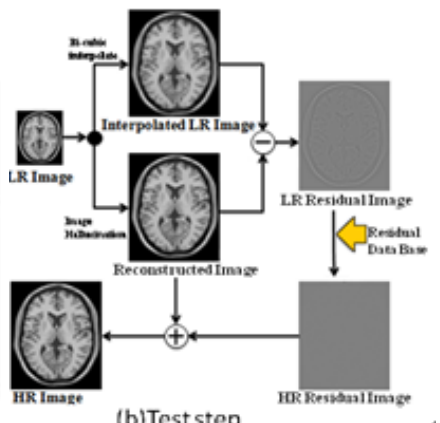

(b) Test step
The grand truth HR image is the left of Figure 4. Others are reconstructed HR images with their PSNR by different methods. It can be seen that the reconstructed high resolution images obtained using our proposed approaches are much better than those obtained by conventional methods.

\section{B. 3D Images}

In medical diagnosis, we can obtain HR axial plane images however we cannot obtain other plane (coronal and sagittal) images satisfy its resolution.Then, we apply the database using axial plane to reconstruct other plane images. In this approach, we obtain HR coronal, sagittal plane images from LR coronal, sagittal plane images using HR axial plane images

In order to validate the effectiveness of our proposed methods, we experiment with our proposed method. We use coronal plane and sagittal plane brain images. The size of each image is $180 \times 180$. We first generate the LR and HR image pairs by down sampling the original images. The size of LR image is $45 \times 45$. In the experiment, the peak signal-to-noise ratio (PSNR)[dB] is used as a quantitative measure for evaluation of the HR reconstruction performance. Our proposed image hallucination method is used with a patch size of $3 \times 3$ and a $1 \times 1$ patch that overlaps with adjacent patches.

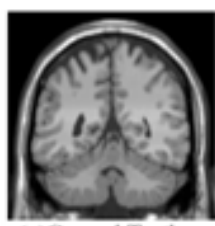

(a)Ground Truth

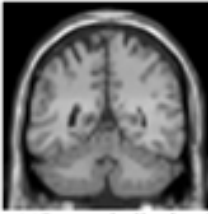

(c)Image hallucination $(28.4546)$

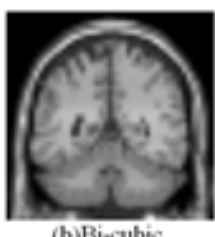

(b)Bi-cubic (25.1771)

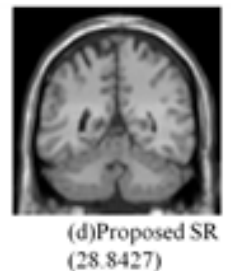

FIGURE V. EXPERIMENTAL RESULTS (CORONAL PLANE).

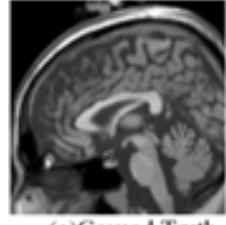

(a)Ground Truth

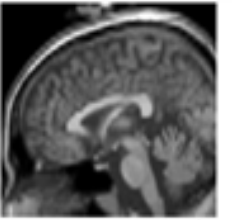

(c)Image Hallucination (25.6078)

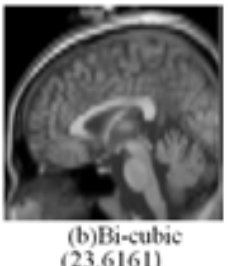

(23.6161)

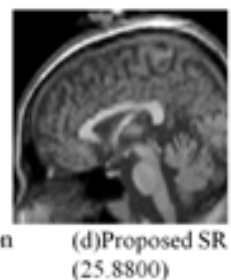

(33.1372)

FIGURE IV. EXPERIMENTAL RESULTS. 
The grand truth HR image is the left of Figure 5, 6. Others are reconstructed HR images with their PSNR by different methods. It can be seen that the reconstructed high resolution images obtained using our proposed approaches are much better than those obtained by conventional methods. The results show that HR coronal, sagittal plane images can be reconstructed LR coronal, sagittal plane images using axial plane images.

\section{CONCLUSIONS}

A new method for high quality MR image reconstructing has been presented that can compensate the high-frequency components of the reconstructed HR image. The basic idea of our proposed residual image compensation framework is to reconstruct or estimate a residual image, which can be used to compensate the high-frequency components of the reconstructed HR image. The proposed method was validated on brain images from brain web. The reconstructed HR image images obtained using our proposed approaches are much better than those obtained by conventional methods. On the problem that coronal or sagittal plane HR images cannot be obtained, we apply the proposed method to reconstruct other plane HR images. Experimental results show that the proposed method can reconstruct HR coronal or sagittal plane by using available LR and HR data pairs in axial plane. In this paper, we propose the new method that improves the reconstruction performance and the new approach that reconstructs HR images using the database that stored data pairs of other plane images. More solid validation on other brain images will be done in future studies.

\section{ACKNOWLEDGEMENT}

This work is supported in part by the Grant-in Aid for Scientific Research from the Japanese Ministry for Education, Science, Culture and Sports (MEXT) under the Grant No. 2430076, No. 24103710, in part by the MEXT Support Program for the Strategic Research Foundation at Private Universities (2013-2017), and in part by the R-GIRO Research Fund from Ritsumeikan University.

\section{REFERENCES}

[1] S. Baker and T. Kanade, "Limits on super-resolution and how to break them", IEEE transactions on Pattern Analysis and Machine Intelligence, vol. 24, no. 9, pp. 1167-1183, 2002

[2] Michal Irani, Shmuel $P$ eleg. "Improving Resolution by Image Registration”, GVGIP: Graphical Models and Image Processing, vol.53, Issue 3, 1991.

[3] S. Baker, T.Kanade: “Super-resolution optical flow”, Technical Report CMU-RI-TR99-36, Carnegie Mellon University, 1999.

[4] W. T. Freeman, T. R. Jones, E. C. Pasztor, "Example-based superresolution”, IEEE Computer Graphics and Applications, vol.22, no.2, pp.56-65, 2002.

[5] H. Chang, D. Y. Yeung, Y. Xiong, "Super-resolution through neighbor embedding", CVPR, vol.1, pp.275-282, 2004.

[6] S. Baker, and T.Kanade, "Hallucination faces" in Proceedings of IEEE International Conference on Automatic Face and Gesture Recognition, March 2000

[7] C. Liu, H.-Y. Shum, and W. T. Freeman, "Face hallucination: Theory and practice." International Journal of Computer Vision, vol 75, no. 1, pp. 115-134, 2007.

[8] X. Wang and X. Tang, "Hallucinating face by eigentransformation”,IEEE Transactions on Systems, Man, and Cybernetics, Part C, vol. 35, no. 3, pp. 425-434, 2005.

[9] Y.-W. Chen, S. Sasatani,X.-H. Han,“Alignment-Free and HighFrequency Compensation in Face Hallucination”, The Scientific World Journal, 2014. 